\title{
Playing Basketball on Wooden and Asphalt Courts-Does Court Surface Affect Foot Loading?
}

\author{
PW Kong*, DZ Nin, RKK Quek and YK Chua
}

Physical Education and Sports Science Academic Group, National Institute of Education, Nanyang Technological University, Singapore

*Corresponding author: Pui W Kong, Associate Professor, Physical Education and Sports Science Academic Group, National Institute of Education, Nanyang Technological University, 1 Nanyang Walk, 637616, Singapore, Tel: +65-62196213, E-mail: puiwah.kong@nie.edu.sg

\begin{abstract}
This study aimed to examine the influence of court surface on foot loading when executing typical basketball tasks. Thirteen male basketball players performed three basketball-related tasks: Layup, jump shot, and maximal effort sprint on wooden and asphalt courts. In-shoe plantar loading was recorded during the basketball movements and peak force (normalised to body weight) was extracted from eight-foot regions. Perceptions of discomfort at the ankle, knee, and back were surveyed using a $10-\mathrm{cm}$ visual analogue scale. Landing from a layup on the wooden court resulted in elevated peak forces at the hallux $(p=0.022)$ and lesser toes $(p=0.007)$ compared with asphalt court. During the sprint acceleration step, higher peak forces were observed at the hallux $(p=0.048)$ and medial forefoot $(p=$ 0.010 ) on wooden court. No difference between court surfaces was found for perception ratings at the ankle, knee, or back. These results suggested that players can experience greater impact forces at the toes and medial forefoot when performing basketball tasks on the more compliant wooden court than asphalt courts.
\end{abstract}

\section{Keywords}

Plantar pressure, Layup, Jump shot, Sprinting

\section{Introduction}

Basketball is one of the most played sports in the world [1]. While professional basketball games are usually played on indoor wooden courts, asphalt-based artificial courts are also popular especially for outdoor settings given the lower maintenance cost and higher durability. Schools are often equipped with both wooden and asphalt courts for physical education (PE) lessons and co-curricular activities. Structural differences exist between the various court surfaces; wooden surfaces consist of a superficial layer of beech wood, while asphalt courts are typically surfaced with a layer of rubber coating for shock absorption [2]. Among coaches, PE teachers and players, landing on wooden courts is generally perceived to be 'softer' with lower impact forces than landing on artificial surfaces. This perception can be partly attributed to the lower stiffness of wooden courts which allows them to undergo a greater extent of deformation upon impact [3]. Although the injury statistics for wooden and artificial playing surfaces in basketball are not known [4], one simulation study has shown that wood resulted in lower landing forces compared to asphalt grounds [2]. The study, however, simulated only vertical landing from $300 \mathrm{~mm}$ and modelled the human body as a rigid lower limb. This simplification fails to take into account the natural joint movements (e.g. knee flexion) that occur during landing. Furthermore, basketball players execute many other impactful movements besides vertical jump landings [5]. Thus, it is necessary to verify the simulation study findings using an experimental approach.

Previous experimental studies found a higher risk of knee (anterior cruciate ligament) injury for female team handball players [6] and traumatic injury for female floorball players [7] on artificial surfaces than wooden floors, possibly influenced by shoe-court friction. The physical demands of basketball are considerably different from those of team handball or floorball, with basketball players being reported to jump approximately 44 times and sprint every $39 \mathrm{~s}$ in a game [5]. Given that 
the foot loading during basketball related movements such as layup and side-cutting are considerably higher than that during running [8], it is crucial to understand the forces acting on players when executing basketball skills on different playing surfaces. Using a force platform, McClay and colleagues [9] quantified the ground reaction forces during typical basketball tasks in professional players and reported elevated forces of up to nine times an individual's body weight upon landing from a jump. To replicate a more realistic playing surface, Nin, Lam and Kong, [10] used a wooden-top force platform to measure impact forces during basketball layup, simulated shot blocking, and drop landing tasks. Comparable force data of such high-impact activities, however, are not available for other artificial playing surfaces commonly used in basketball.

While traditional force platforms are useful to quantify the total ground reaction forces [9-11], they are often limited to laboratory settings and unable to locate regional load at specific parts of the foot. Recently, there has been increasing use of mobile in-shoe plantar measurement systems to gain insights into the foot loading when executing sports tasks on various playing surfaces. For example, Ford, et al. [12] compared the in-shoe loading patterns during cutting on natural grass and synthetic turf among male football players. Similarly, Tessutti, Ribeiro, Trombini-Souza, and Sacco, [13] examined foot pressure during running on four different surfaces: Asphalt, concrete, rubber, and natural grass. Although several studies have reported plantar pressure data on basketball-related tasks [8,14-19], to our best knowledge, the influence of basketball court surfaces on foot loading remains unknown.

The purpose of this study was, therefore, to investigate the influence of court surface on foot loading during typical basketball tasks using an experimental approach. It was hypothesized that lower forces, measured using an in-shoe system, would be observed on wooden courts compared with asphalt courts.

\section{Methods}

\section{Participants}

Based on simulation results reported by Kim, et al. [2], a very large difference in peak ground reaction force between asphalt and wood surfaces were found. Thus, a large effect size of 0.8 was used in a power analysis to determine the minimum sample size of 12 ( $\alpha=0.05$, power $=0.80$, one-tail). To account for potential dropout and technical errors, thirteen healthy basketball players (age $=23.0(1.4)$ years, height $=1.75(0.05) \mathrm{m}$, mass $=68.4(8.6) \mathrm{kg}$ ) were recruited for the study. The inclusion criteria were 1) Males; 2) University students who participated in the Institute Inter-hall Basketball Games; 3) Had more than five years of recreational basketball experience, and 4) Had foot size of US 9.0 or 11.0 measured by a Brannock device. All participants were free from any lower-extremity injuries for six months prior to the time of study. The procedures were approved by the Nanyang Technological University Institutional Review Board. Participants were informed about the experimental procedures, potential benefits and risks, and their rights to withdraw at any point of the study. Prior to testing, written consent were obtained from all participants.

\section{Procedures}

The experiment took place in an indoor wooden basketball court and an outdoor asphalt court coated with All Sport surface (California Products Corporation, Andover, USA). The Novel Pedar-X system (Novel GmbH, Munich, Germany) with 99 sensors within each insole was used to measure plantar forces. Two pairs of insoles, US size 9.0 and 11.0 , were calibrated to $700 \mathrm{kPa}$ with the Trublu calibration device (Novel $\mathrm{GmbH}$, Munich, Germany) according to the manufacturer's guidelines. To avoid the influence of footwear, the same make and model of basketball shoes (US size 9.0 or 11.0, Nike Black Mumba 24, Portland, USA) were used across all participants. Participants also wore a new pair of socks provided by the researchers and used the same ball ( $\mathrm{Li}$ Ning B6000, Beijing, China) for all shooting tasks.

Eligible participants reported for experimental testing on one occasion. First, they were surveyed on their playing preferences and habits on wooden and artificial courts. Next, the wireless Pedar- $X$ device with the insoles was attached to the participants. After five minutes of warm-up using their own routines, participants proceeded to an assigned basketball court (wooden or asphalt, presented in a randomized order) for familiarization with the test tasks. Three typical basketball-related tasks were selected: 1) Layup; 2) Jump shot, and 3) Sprint (Figure 1). In-shoe plantar loading was recorded at $100 \mathrm{~Hz}$ while performing these tasks.

Layup (Figure 1a): The layup is the most commonly employed technique for scoring during basketball games [20], and the landing biomechanics of this task has been evaluated in numerous studies [14-17]. For consistency, dribbling was prohibited prior to the runup and a right-handed layup was performed by all participants $[10,18,19]$. A recent study showed that for basketball layup tasks, a minimum of six to eight trials were needed to obtain stable peak force of the whole foot using the Pedar-X system [18]. To ensure that the peak force data collected were sufficiently reliable, 10 valid trials were recorded in the present study. A trial being considered valid if (i) The shot was successful; (ii) The ball made contact with the rim; or (iii) The ball made contact with both the back-board and the rim.

Jump shot (Figure 1b): The jump shot is identified as an effective and frequently used shooting technique, receiving much attention in biomechanical studies $[17,21]$. Participants were tasked to perform jump shots 
a) Layup. A: Starting position and initiation with right forward step; B: Second contralateral step and takeoff with left leg; C: Flight phase and release of ball into goal; D: Landing.

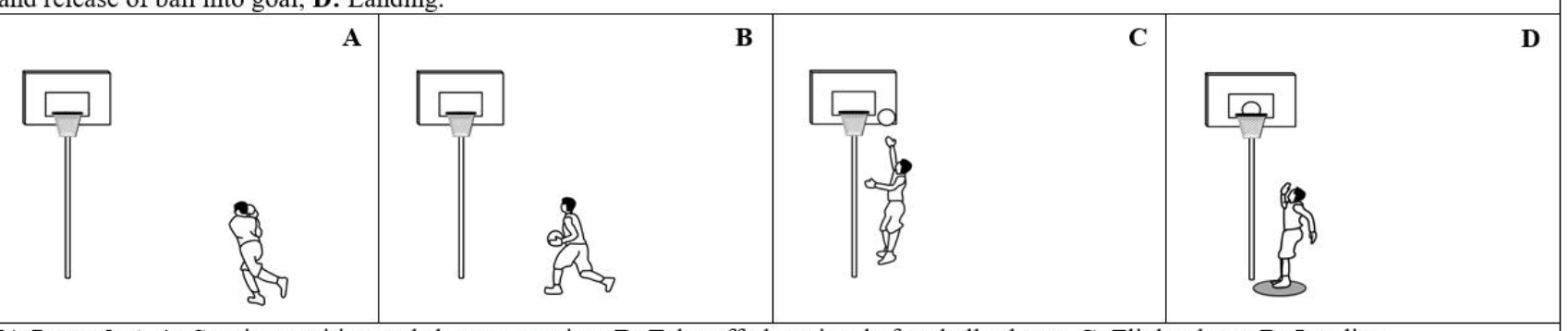

b) Jumpshot. A: Starting position and shot preparation; B: Take-off phase just before ball release; C: Flight phase; D: Landing.

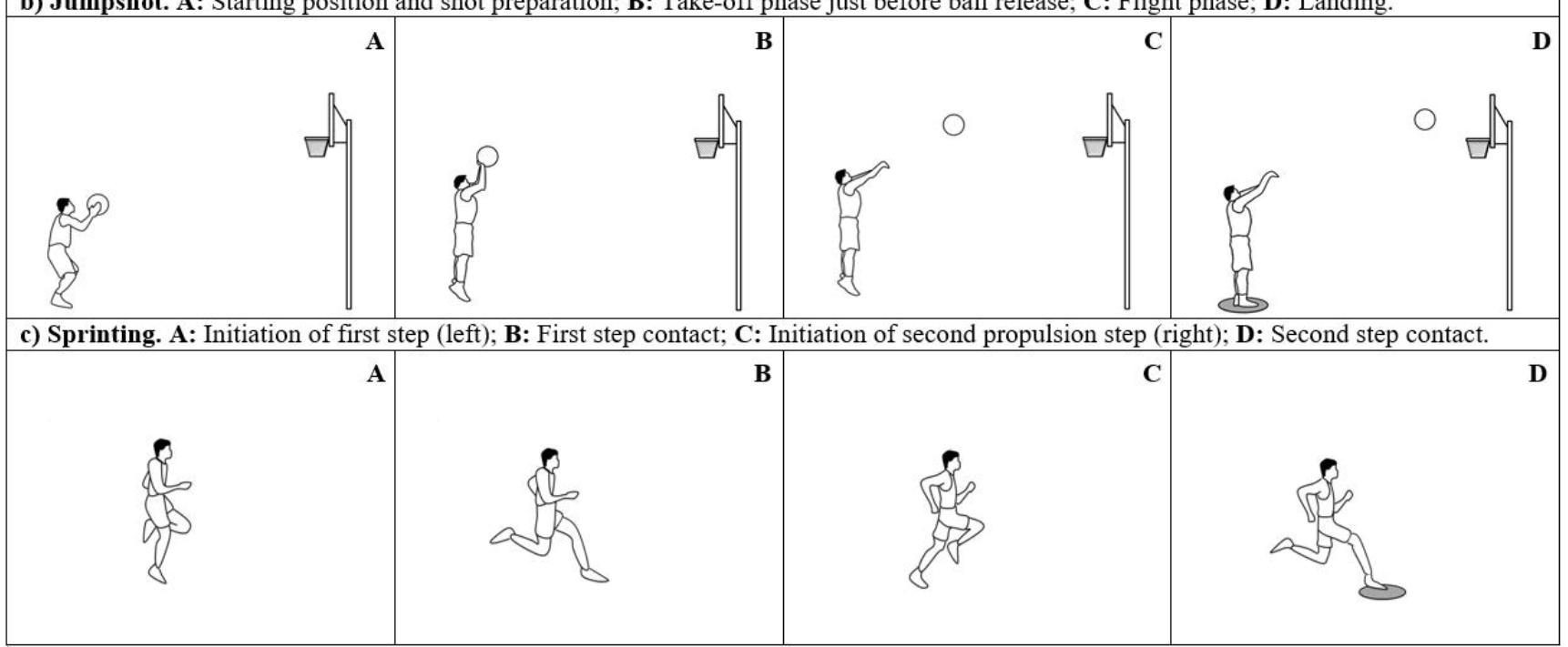

Figure 1: Sequences of three typical basketball-related tasks: a) Layup; b) Jump shot; and c) Maximum forward sprint. Grey circle indicates the step selected for analysis in each task.

at the free throw line using their preferred arm. All except one participant shot with the right arm. Ten valid trials were recorded using the same criteria as for the layup described previously.

Sprint (Figure 1c). Maximal forward sprinting is highly relevant to basketball since players sprint approximately every $39 \mathrm{~s}$ in a game [5]. Participants sprinted at maximal effort across the court, using the left foot as the first step. Since the sprint task was performed at maximal effort and hence more demanding than the other tasks, five instead of 10 successful trials were recorded as done in a previous study on basketball-related movements [8].

After completing all tasks on one court surface (wooden or asphalt), participants were asked to rate their perceived level of discomfort at their ankles, knees, and back using a visual analogue scale (VAS). The VAS ranged from 0 (No discomfort) to $10 \mathrm{~cm}$ (Worst possible discomfort) and was measured to the nearest $0.1 \mathrm{~cm}$. The same in-foot loading measurements and subjective perception procedures were then repeated for the other court condition (wooden or asphalt).

\section{Data processing}

The double-leg landing steps of the layup and jump shot were analyzed (Figure 1a and Figure 1b). Data of the left and right feet were arranged into the shooting and non-shooting side. The shooting side was defined as the foot on the same side of the shooting arm (right $n=$ 12 , left $n=1$ ). For the sprint, the second (right) step representing the acceleration phase was chosen for analysis $[8,19,22]$ (Figure 1c). A mask of the eight regions (hallux, lesser toes, medial, central and lateral forefoot, medial and lateral arch, and heel) created using the Novel Multimask software (Novel GmbH, Munich, Germany) was applied to extract the peak force in each foot region (Figure 2). The peak force indicates the maximal force in one-foot region during one step, and this variable is commonly used in other studies examining plantar loading during basketball-related tasks [14-18]. Peak forces were then normalised to participants' body weight (BW). An average value of all valid trials for each movement was used for subsequent analysis.

\section{Statistical analysis}

Statistical analyses were performed using SPSS Version 21.0 (IBM Corp, Armonk, NY, USA), with significance was set at $P<0.05$. Data are expressed in mean (standard deviation). For the layup and jump shot landings, analysis of variance (ANOVA) with repeated measures (Side $\times$ Court) was applied to the peak forces in each of the eight foot regions. To correct for violation of sphericity, significance was assessed from the Greenhouse-Geisser correction for epsilon values $\leq 0.75$, and the Huynh-Feldt correction for epsilon $>0.75$. Effect size (partial eta squared, $\eta_{p}{ }^{2}$ ) was calculated to describe the magnitude of the difference and values of 0.01, 0.09 and 


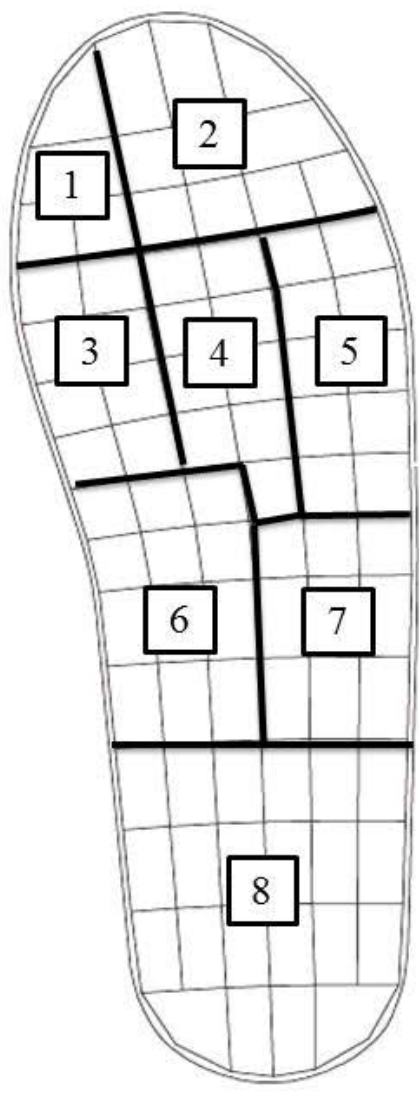

1- Hallux

2- Lesser Toes

3- Medial Forefoot

4- Central Forefoot

5- Lateral Forefoot

6- Medial Arch

7- Lateral Arch

8- Heel

Figure 2: The eight-region mask for data extraction of in-shoe foot loading measurements.

0.25 were interpreted as small, medium and large effects, respectively [23]. Should a significant Side $\times$ Court interaction be found, post-hoc pairwise comparisons with Bonferroni adjustment were applied.

For VAS ratings and sprint acceleration peak forces, Wilcoxon signed-rank test was used to compare between the wooden and asphalt courts data. Non-parametric test was chosen owing to the relatively small sample size. Effect size $(r)$ was calculated and interpreted as follows: Small $0.1 \leq|r| \leq 29$, medium $0.3 \leq|r| \leq$ 0.49 , and large $|r| \geq 0.5$ [24].

\section{Results}

For the layup landing, elevated peak forces were found on the wooden court than the asphalt court in two-foot regions (Table 1): Hallux $(P=0.022$, large effect size) and lesser toes ( $P=0.007$, large effect size). There were a few bilateral differences of large effect sizes between the shooting and non-shooting sides. For the only significant Side $\times$ Court interaction observed in the medial forefoot $(P=0.036$, large effect size, Table $1)$, post-hoc analysis showed significant side-to-side difference on the asphalt court but not the wooden court. During jump shot landing, there was no main effect of the court type or Side $\times$ Court interaction (Table 2). As shown in Table 2, only significant bilateral differences were found, with the non-shooting side displaying higher forces than the shooting side at the medial forefoot $(P$ $=0.039$, large effect size $)$ and the heel $(P=0.002$, large effect size). During the sprint acceleration step, higher peak forces were observed on the wooden court compared to asphalt court in two regions: hallux $(P=0.048$, medium effect size) and medial forefoot $P=0.010$, large effect size, Table 3).

Eight out of thirteen (61.5\%) participants generally preferred to play on wooden than artificial courts. After performing the three basketball-related tasks on both surfaces, no significant differences were found in the VAS ratings at the ankle (wooden $=0.92(1.11) \mathrm{cm}$, asphalt $=1.05(0.99) \mathrm{cm}, P=0.635, r=-0.09)$, knee (wooden $=1.13(1.68) \mathrm{cm}$, asphalt $=1.46(1.64) \mathrm{cm}, P=0.293$, $r=-0.21$ ), and back (wooden $=0.89(1.25) \mathrm{cm}$, asphalt $=$ $0.94(0.93) \mathrm{cm}, P=0.929, r=-0.02)$.

\section{Discussion}

This study investigated the influence of court surface (wood and asphalt) on foot loading during three basketball-specific manoeuvres. Contrary to our hypothesis that lower forces would be observed on wooden than asphalt court, our findings showed that wooden court resulted in higher peak forces at the toes and medial forefoot during layup landing and sprinting. These findings oppose the common beliefs by coaches, PE teachers and athletes that wooden courts can provide better force attenuation compared to asphalt courts.

\section{Landing from jumps}

Although a wooden court presents a softer landing 
Table 1: Statistical results of peak forces (in body weight) in eight foot regions during layup landing.

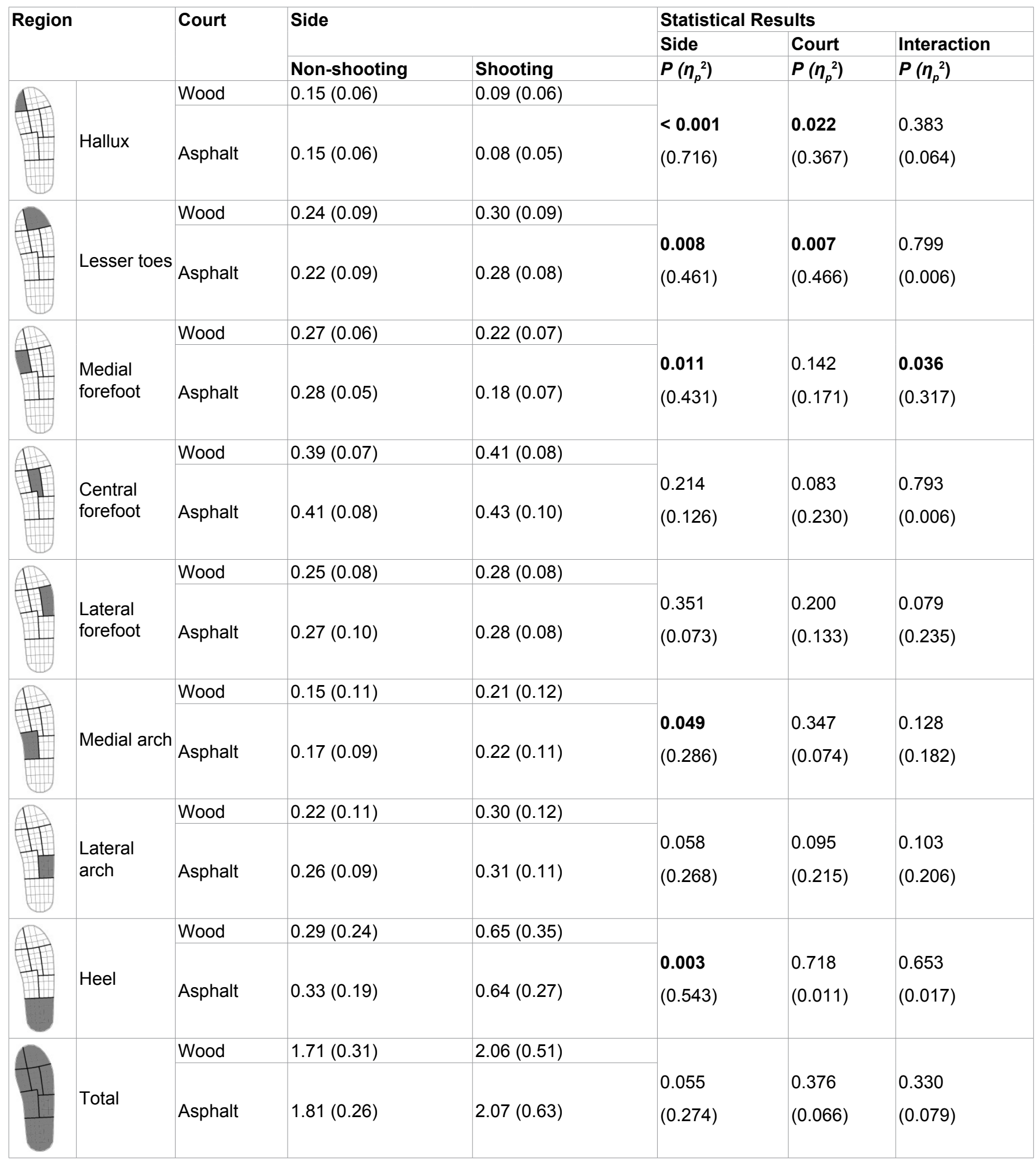

Note: Data are expressed in mean (SD). The shooting side was defined as the foot on the same side of the shooting arm (right for all participants). Significant $P$-values from repeated measures ANOVA $(P<0.05)$ are shown in bold. Effect size $\left(\eta_{p}^{2}\right)$ values of $0.01,0.09$ and 0.25 were interpreted as small, medium and large effects, respectively.

surface [3], the present study showed that force attenuation was less effective on the wooden than asphalt court when landing from a layup. This is in contrast to the results obtained from a simulation study conducted by Kim, and colleagues [2], which demonstrated that wood ground produced lower peak forces than asphalt. It is believed that the simulation study over-simplified the human body as a rigid lower limb with no flexion/ extension movement abilities. In reality, participants are likely to adopt slightly different landing techniques in response to the landing surface [25]. The present study allowed players to perform typical basketball tasks on real courts, providing good ecological validity over mechanical tests, simulation studies, and controlled laboratory experiments (e.g. drop landing on a force platform). Our findings are consistent with previous experimental studies in which higher peak vertical forces were associated with landing onto a mat compared to a non-mat condi- 
Table 2: Statistical results of peak forces (in body weight) in eight foot regions during jump shot landing.

\begin{tabular}{|c|c|c|c|c|c|c|c|}
\hline \multirow{2}{*}{\multicolumn{2}{|c|}{ Region }} & \multirow{4}{*}{$\begin{array}{l}\text { Court } \\
\text { Wood }\end{array}$} & \multirow{2}{*}{\multicolumn{2}{|c|}{ Side }} & \multirow{2}{*}{\multicolumn{3}{|c|}{ Statistical Results }} \\
\hline & & & & & & & \\
\hline & & & \multirow{2}{*}{$\begin{array}{l}\text { Non-shooting } \\
0.12(0.06)\end{array}$} & \multirow{2}{*}{$\begin{array}{l}\text { Shooting } \\
0.08(0.04)\end{array}$} & $\begin{array}{l}\text { Side } \\
P\left(\eta_{p}^{2}\right)\end{array}$ & $\begin{array}{l}\text { Court } \\
P\left(\eta_{p}^{2}\right)\end{array}$ & $\begin{array}{l}\text { Interaction } \\
\boldsymbol{P}\left(\boldsymbol{\eta}_{p}^{2}\right)\end{array}$ \\
\hline & \multirow[b]{2}{*}{ Hallux } & & & & \multirow[b]{2}{*}{$\begin{array}{l}0.073 \\
(0.244)\end{array}$} & \multirow[b]{2}{*}{$\begin{array}{l}0.906 \\
(0.001)\end{array}$} & \multirow[b]{2}{*}{$\begin{array}{l}0.240 \\
(0.113)\end{array}$} \\
\hline & & Asphalt & $0.11(0.07)$ & $0.09(0.05)$ & & & \\
\hline & \multirow[b]{2}{*}{ Lesser toes } & Wood & $0.21(0.10)$ & $0.22(0.10)$ & \multirow[b]{2}{*}{$\begin{array}{l}0.432 \\
(0.052)\end{array}$} & \multirow[b]{2}{*}{$\begin{array}{l}0.283 \\
(0.095)\end{array}$} & \multirow[b]{2}{*}{$\begin{array}{l}0.782 \\
(0.007)\end{array}$} \\
\hline & & Asphalt & $0.18(0.10)$ & $0.21(0.11)$ & & & \\
\hline & \multirow[b]{2}{*}{$\begin{array}{l}\text { Medial } \\
\text { forefoot }\end{array}$} & Wood & $0.25(0.10)$ & $0.18(0.09)$ & \multirow[b]{2}{*}{$\begin{array}{l}0.039 \\
(0.309)\end{array}$} & \multirow[b]{2}{*}{$\begin{array}{l}0.820 \\
(0.004)\end{array}$} & \multirow[b]{2}{*}{$\begin{array}{l}0.268 \\
(0.111)\end{array}$} \\
\hline & & Asphalt & $0.24(0.10)$ & $0.20(0.09)$ & & & \\
\hline & \multirow[b]{2}{*}{$\begin{array}{l}\text { Central } \\
\text { forefoot }\end{array}$} & Wood & $0.40(0.11)$ & $0.34(0.23)$ & \multirow[b]{2}{*}{$\begin{array}{l}0.260 \\
(0.105)\end{array}$} & \multirow[b]{2}{*}{$\begin{array}{l}0.535 \\
(0.033)\end{array}$} & \multirow[b]{2}{*}{$\begin{array}{l}0.673 \\
(0.015)\end{array}$} \\
\hline & & Asphalt & $0.36(0.10)$ & $0.32(0.13)$ & & & \\
\hline & \multirow[b]{2}{*}{$\begin{array}{l}\text { Lateral } \\
\text { forefoot }\end{array}$} & Wood & $0.23(0.09)$ & $0.20(0.12)$ & \multirow[b]{2}{*}{$\begin{array}{l}0.568 \\
(0.028)\end{array}$} & \multirow[b]{2}{*}{$\begin{array}{l}0.580 \\
(0.026)\end{array}$} & \multirow[b]{2}{*}{$\begin{array}{l}0.227 \\
(0.119)\end{array}$} \\
\hline & & Asphalt & $0.20(0.07)$ & $0.21(0.11)$ & & & \\
\hline & \multirow[b]{2}{*}{ Medial arch } & Wood & $0.07(0.07)$ & $0.05(0.06)$ & \multirow[b]{2}{*}{$\begin{array}{l}0.751 \\
(0.015)\end{array}$} & \multirow[b]{2}{*}{$\begin{array}{l}0.185 \\
(0.142)\end{array}$} & \multirow[b]{2}{*}{$\begin{array}{l}0.115 \\
(0.194)\end{array}$} \\
\hline & & Asphalt & $0.07(0.07)$ & $0.08(0.10)$ & & & \\
\hline & \multirow[b]{2}{*}{$\begin{array}{l}\text { Lateral } \\
\text { arch }\end{array}$} & Wood & $0.16(0.10)$ & $0.10(0.08)$ & \multirow[b]{2}{*}{$\begin{array}{l}0.192 \\
(0.139)\end{array}$} & & \\
\hline & & Asphalt & $0.15(0.06)$ & $0.13(0.10)$ & & $\begin{array}{l}0.584 \\
(0.026)\end{array}$ & $\begin{array}{l}0.091 \\
(0.220)\end{array}$ \\
\hline & & Wood & $0.38(0.24)$ & $0.23(0.23)$ & & & \\
\hline & Heel & Asphalt & $0.35(0.18)$ & $0.22(0.17)$ & $\begin{array}{l}0.002 \\
(0.564)\end{array}$ & $\begin{array}{l}0.728 \\
(0.010)\end{array}$ & $\begin{array}{l}0.957 \\
(<0.001)\end{array}$ \\
\hline & & Wood & $1.30(0.40)$ & $1.09(0.58)$ & & & \\
\hline & Total & Asphalt & $1.23(0.26)$ & $1.20(0.53)$ & $\begin{array}{l}0.483 \\
(0.042)\end{array}$ & $\begin{array}{l}0.834 \\
(0.004)\end{array}$ & $\begin{array}{l}0.244 \\
(0.111)\end{array}$ \\
\hline
\end{tabular}

Note: Data are expressed in mean (SD). The shooting side was defined as the foot on the same side of the shooting arm (right $\mathrm{n}=12$, left $\mathrm{n}=1)$. Significant $P$-values from repeated measures ANOVA $(P<0.05)$ are shown in bold. Effect size $\left(\eta_{p}^{2}\right)$ values of $0.01,0.09$ and 0.25 were interpreted as small, medium and large effects, respectively.

tion $[25,26]$. It is possible that participants in the present study adopted a stiffer landing strategy owing to the perceived higher compliance of the wooden compared to asphalt surface. The higher force experienced when landing on a more compliant surface is due to a stiffer landing strategy characterized by reduced hip and knee joint flexion, coupled with increased activation of muscles crossing the knee joint [25]. Future studies can confirm this speculation by including kinematic variables.

\section{Sprint acceleration}

It was found that when sprinting on the wooden court, participants pushed off with greater force at the hallux and medial forefoot compared to when moving across the asphalt court. While it is possible that participants required more forces to push off from a more compliant surface, the adoption of a different sprint technique (increased planting at the forefoot) is likely to be part of a compensatory mechanism to augment 
Table 3: Statistical results of peak forces (in body weight) in eight foot regions during sprint acceleration step.

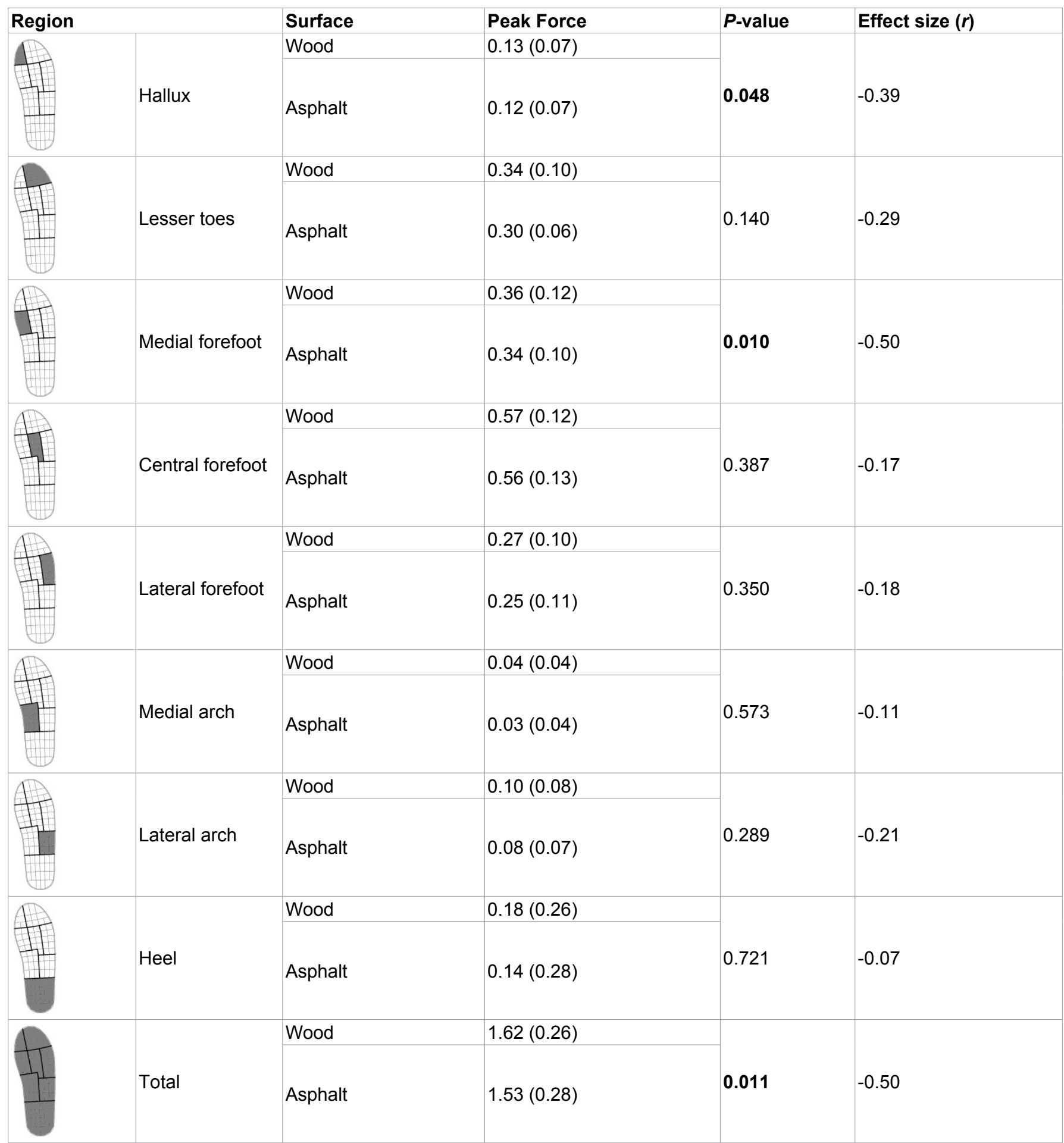

Note: Data are expressed in mean (SD). Significant $P$-values from Wilcoxon signed-rank tests $(P<0.05)$ are shown in bold. Effect sizes were interpreted as: small $0.1 \leq|r| \leq 29$, medium $0.3 \leq|r| \leq 0.49$, large $|r| \geq 0.5$.

shoe-court friction on the wooden court. Wooden surfaces have been found to possess friction coefficients which are less than half of those of their asphalt counterparts [27]. Thus, it was probable that participants executed a sprinting technique which induced greater traction, in order to reduce the likelihood of slipping and its resultant injury. However, it is also essential to acknowledge that excessive shoe-court friction might give rise to lower extremity injuries caused by overloading [28]. Moreover, increased regional loading at the foot could lead to higher skin temperature which could in turn result in blistering [29].

\section{Potential role of footwear}

Both the layup and sprint are common movements in basketball; the effect of increased peak forces experienced while executing these manoeuvres on the wooden court is amplified due to the frequency at which they are performed. Players, PE teachers and coaches should be mindful of the loading demands across different shoe-court combinations, in particular, the increased forces associated with a more compliant and slippery surface. Although the playing environment is usually unmodifiable, wearing appropriate footwear can play an important role in attenuating forces. Most studies inves- 
tigating the interaction between athletic shoe and playing surface have focused on the property of shoe-surface traction $[30,31]$. The findings of this study suggest that shoe-surface interaction can also affect vertical impact loading alongside shearing forces. Thus, shoe cushioning properties such as midsole hardness should be investigated along with frictional properties to provide a better understanding of the shoe-surface interaction. This is especially imperative for sports such as basketball which frequently involves both jumping and running movements. Future work could look at how both the frictional and cushioning properties of a shoe influence contact forces at foot-shoe and shoe-surface interfaces.

\section{Perceptual response to playing surfaces}

In addition to biomechanical loadings, perceptual responses of the participants to landing on the different surfaces were also studied. It was found that participants perceived landing on both surfaces to be equally comfortable. A previous study showed that basketball players are able to distinguish between shoe midsole hardness conditions through the perceptual parameter of comfort level while performing several basketball movements [10]. In another study on layup and side-cutting tasks, recreational basketball players indicated similar perceived stability for shoes with softer and harder midsoles, and that there was no relationship between biomechanical and subjective measurements [32]. In the present study, the majority of participants preferred playing on a wooden to an asphalt court. There were, however, no differences in perceptual responses to comfort at the ankle, knee, and back after performing basketball-related movements on both courts. This suggests that players might be more sensitive to changes in shoe hardness $[10,19]$ compared to surface compliance. It is also likely that a certain threshold of impact force may be required for neural feedback of the body before an individual can accurately differentiate between shoe-surface compliance conditions. Given that players' court preference can be influenced by factors other than comfort, future studies should consider investigating the relationship between perceived surface compliance and landing biomechanics.

\section{Bilateral asymmetry}

The layup and jump shot are movements involving a double-leg landing; the bilateral asymmetry of such landings has been found to be associated with lower-limb injuries [33]. An interesting secondary finding in the present study showed that when landing from a layup, side-to-side asymmetry of impact forces exists, with substantial asymmetry directed towards the shooting side at the lateral arch and heel regions. This bilateral asymmetry might have developed from prolonged participation in a sport which relies predominantly on unilateral upper-limb movements, for example, dribbling and shooting in basketball. It is possible that in the kinetic chain of different basketball movements, an ex- tensive unilateral upper-limb usage had an imbalanced strengthening effect on the lower-limbs, thereby resulting in asymmetrical landing strategies. As such, players and coaches should be aware of the loading asymmetry when landing from these movements and implement appropriate interventions such as using customised orthoses. Future research should consider examining the effect of upper-limb dominance on lower-limb biomechanics during basketball tasks.

\section{Limitations}

There were a few limitations to this study. Firstly, no kinematic or performance variables such as jump height and sprint speed were obtained. Such information would have been useful in understanding the relationship between surface compliance and movement technique adopted by the participants. Considering that the participants in the present study were skilled players and that the movement tasks studied were basic and frequently executed skills $[5,17,20,21]$, it is unlikely that they would alter their performance substantially due to surface compliance. Moving forward, kinematic analysis should be included in addition to foot loading such that jump height and landing techniques between surfaces can be compared. Secondly, mechanical tests to accurately measure surface compliance and shoe-surface traction were not conducted due to the constraints in facilities and resources in our laboratory. Since the wooden and asphalt surfaces used in the present study are standard sport courts, it is expected that their respective mechanical properties are similar to those reported in the literature. To strengthen the study design, future studies should include mechanical tests to measure the stiffness of different court surfaces. Thirdly, only peak forces at the foot were measured and these forces do not necessarily reflect the loading at individual joints such as the knee and the hip. Inverse dynamics calculations would be needed to quantify joint kinetics for a more comprehensive analysis. Finally, it should be acknowledged the $P$-values reported throughout are unadjusted nominal values. Since the peak force in several foot regions were statistically compared, readers should be aware of the increased change of committing type I error resulting from multiple comparisons.

\section{Conclusion}

As opposed to common perception and previous simulation study findings, the present experimental study on basketball players showed that wooden courts did not provide better impact force attenuation compared to asphalt courts. Instead, players experienced greater peak forces at the toes and medial forefoot on the more compliant wooden court during layup landing and sprinting. Coaches, PE teachers and athletes should be informed that playing basketball on wooden courts can expose players to higher forces in the foot. Future studies should investigate the interplay between playing surface, foot loading, and risk of injuries. 


\section{Disclosure of Interest}

The authors declare that they have no competing interest.

\section{References}

1. Daneshvar DH, Nowinski CJ, McKee AC, Cantu RC (2011) The epidemiology of sport-related concussion. Clin J Sports Med 30: 1-17.

2. Kim SH, Cho JR, Choi JH, Ryu SH, Jeong WB (2012) Coupled foot-shoe-ground interaction model to assess landing impact transfer characteristics to ground condition. Interaction and Multiscale Mechanics 5: 75-90.

3. Bartlett R, Gratton C, Rolf C (2006) Encyclopedia of International Sports Studies. Routledge, London.

4. Dragoo JL, Braun HJ (2010) The effect of playing surface on injury rate. Sports Med 40: 981-990.

5. Ben Abdelkrim N, El Fazaa S, El Ati J (2007) Time-motion analysis and physiological data of elite under-19-year-old basketball players during competition. Br J Sports Med 41: 69-75.

6. Olsen OE, Myklebust G, Engebretsen L, Holme I, Bahr R (2003) Relationship between floor type and risk of acl injury in team handball. Scand J Med Sci Sports 13: 299-304.

7. Pasanen K, Parkkari J, Rossi L, Kannus P (2008) Artificial playing surface increases the injury risk in pivoting indoor sports: A prospective one-season follow-up study in finnish female floorball. Br J Sports Med 42: 194-197.

8. Kong PW, Lam WK, Ng WX, Aziz L, Leong HF (2018) Inshoe plantar pressure profiles in amateur basketball players-implications for footwear recommendations and orthosis use. J Am Podistr Med Assoc 108: 215-224.

9. McClay IS, Robinson JR, Andriacchi TP, Frederick EC, Gross T, et al. (1994) A profile of ground reaction forces in professional basketball. J Appl Biomech 10: 222-236.

10. Nin DZ, Lam WK, Kong PW (2016) Effect of body mass and midsole hardness on kinetic and perceptual variables during basketball landing manoeuvres. J Sports Sci 34: 756-765.

11. Zhang S, Clowers K, Kohstall C, Yu YJ (2005) Effects of various midsole densities of basketball shoes on impact attenuation during landing activities. J Appl Biomech 21: 3-17.

12. Ford KR, Manson NA, Evans BJ, Myer GD, Gwin RC, et al. (2006) Comparison of in-shoe foot loading patterns on natural grass and synthetic turf. J Sci Med Sport 9: 433-440.

13. Tessutti V, Ribeiro AP, Trombini-Souza F, Sacco IC (2012) Attenuation of foot pressure during running on four different surfaces: Asphalt, concrete, rubber, and natural grass. J Sports Sci 30: 1545-1550.

14. Guettler JH, Ruskan GJ, Bytomski JR, Brown CR, Richardson JK, et al. (2006) Fifth metatarsal stress fractures in elite basketball players: Evaluation of forces acting on the fifth metatarsal. Am J Orthop 35: 532-536.

15. Yu B, Lin CF, Garrett WE (2006) Lower extremity biomechanics during the landing of a stop-jump task. Clin Biomech 21: 297-305.
16. Queen RM, Mall NA, Nunley JA, Chuckpaiwong B (2009) Differences in plantar loading between flat and normal feet during different athletic tasks. Gait Posture 29: 582-586.

17. Pau M, Ciuti C (2013) Stresses in the plantar region for long- and short-range throws in women basketball players. Eur J Sport Sci 13: 575-581.

18. Chua YK, Quek RK, Kong PW (2017) Basketball lay-up-foot loading characteristics and the number of trials necessary to obtain stable plantar pressure variables. Sports Biomech 16: $13-22$.

19. Lam WK, Ng WX, Kong PW (2017) Influence of shoe midsole hardness on plantar pressure distribution in four basketball-related movements. Res Sports Med 25: 37-47.

20. Wang J, Liu W, Moffit J (2009) Skills and offensive tactics used in pick-up basketball games. Percept Mot Skills 109: 473-477.

21. Okazaki VH, Rodacki AL, Satern MN (2015) A review on basketball jump shot. Sports Biomech 14: 190-205.

22. Coh M, Tomazin K, Stuhec S (2006) The biomechanical model of the sprint starts and block acceleration. Facta Universitatis Series Physical Education and Sport 4: 103-114.

23. Hanna D, Dempster M (2012) Psychology Statistics for Dummies. John Wiley and Sons, West Sussex, 165-183.

24. Field A (2009) Discovering Statistics Using SPSS. ( $3^{\text {rd }}$ edn), SAGE Publication, London, 56-57.

25. http://www.asbweb.org/conferences/2012/abstracts/225. pdf

26. McNitt-Gray JL, Yokoi T, Millward C (1994) Landing strategies used by gymnasts on different surfaces. J Appl Biomech 10: 237-252.

27. Carre MJ, Clarke JD, Damm L, Dixon SJ (2014) Friction at the tennis shoe-court interface: How biomechanically informed lab-based testing can enhance understanding. Procedia Engineering 72: 883-888.

28. Wannop JW, Worobets JT, Stefanyshyn DJ (2010) Footwear traction and lower extremity joint loading. Am J Sports Med 38: 1221-1228.

29. Yavuz M, Davis BL (2010) Plantar shear stress distribution in athletic individuals with frictional foot blisters. J Am Podiatr Med Assoc 100: 116-120.

30. Bentley JA, Ramanathan AK, Arnold GP, Wang W, Abboud RJ (2011) Harmful cleats of football boots: A Biomechanical Evaluation. Foot Ankle Surgery 17: 140-144.

31. Grund T, Senner V (2010) Traction behavior of soccer shoe stud designs under different game-relevant loading conditions. Procedia Engineering 2: 2783-2788.

32. Leong HF, Lam W, Ng WX, Kong PW (2018) Centre of pressure and perceived stability in basketball shoes with soft and hard midsoles. J Appl Biomech 3: 1-7.

33. Hewett TE, Myer GD, Ford KR, Heidt RS Jr, Colosimo AJ, et al. (2005) Biomechanical measures of neuromuscular control and valgus loading of the knee predict anterior cruciate ligament injury risk in female athletes: A prospective study. Am J Sports Med 33: 492-501. 